\title{
3D-form metrology of arbitrary optical surfaces by absorption in fluids
}

\author{
Juan Carlos Martínez Antón, Juan Manuel Plaza Ortega, José Alonso \\ Facultad de Óptica y Optometría, UCM., C/ Arcos de Jalón 118, 28037 Madrid, SPAIN.
}

*jcmartin@fis.ucm.es; phone 34 - 91394 6906; fax 34 - 91394 6885;

\begin{abstract}
We present an imaging technique for the 3D-form metrology of optical surfaces. It is based on the optical absorption in fluids situated between the surface and a reference. An improved setup with a bi-chromatic light source is fundamental to obtain reliable topographic maps. It is able to measure any surface finish (rough or polished), form and slope and independently of scale. We present results focused on flat and spherical optical surfaces, arrays of lenses and with different surface finish (rough-polished). We achieve form accuracies from several nanometers to sub-lambda for sag departures from tens to hundred of microns. Therefore, it seems suitable for the quality control in the production of precision aspheric, freeform lenses and other complex shapes on transparent substrates, independently of the surface finish.
\end{abstract}

Keywords: Optical surface metrology, TOPAF, profilometry, surface finish, ground surface, aspherics, free-form, steep slopes.

\section{INTRODUCTION}

Accurate profilometry of optical surfaces is of increasing importance and difficulty as the production of optical components expands its capabilities and the applications and the surface topologies are more complex. This is the case for aspheric and free-form lenses, Fresnel optics, micro-optical arrays [1], etc... There are numerous non-contact optical methods to determine the topography of surfaces. Most of them use the reflection of light on the measured surface and they are mainly based on interferometry [2-4], confocal profilometry [5-6] and structured light projection and triangulation techniques [7-8]. However, for most conventional approaches, a moderate surface slope or a quick sag departure is difficult to handle. Different aspects are behind this fact, being the more basic that the light is lost when reflection occurs at increasing surface slopes but not only. For high precision optics, interferometry is the gold standard approach. Even so, it cannot see mild slopes or sag departures due to the high fringe density that appears in the interferometric images.

We propose an optical profilometric method that works with transmitted light and is based on the optical absorption of a dye in a fluid solution, which gauges height variations. The use of transmitted light through liquids has already been used for the optical determination of surface topography [9-15]. In the work of Purcell et al [9], still based on interferometry, a non-absorbing liquid is used to reduce optical path differences at steep slopes by index matching with the surface materials. This allows the use of known interference techniques to explore micro-prisms and other steep surface profiles [9]. Typically they need a tight control of refractive index values to adapt to different situations. On the other hand, we find in the literature the use of optical absorption to estimate depth or thickness [10-15]. Although the Lambert-Beer law is commonly used to calculate the specific absorption of substances [10-11], it can be seen the other way around, i.e., to estimate the thickness of solid films [12], or to obtain the relative profile of a surface compared to a reference surface. In this last case, a liquid with known absorption fills the space between both elements [13-15]. For this strategy to be useful, a reference optical signal is necessary to estimate transmittance. In the work of Ogilvie et al [13-14] this is accomplished by using clear water instead of the dye solution in the same setup. This approach is cumbersome and the procedure is prone to misalignment, dye contamination and other errors. Model et al [15] implicitly work under the assumption of constant radiance over the surface. This is difficult to achieve experimentally as we will see and, in their setup, the aperture limited illumination may lead to vignetting for steep surfaces, where again, light may be lost by refraction and therefore it leads to profile errors. Furthermore, none of the previous works really tackle the metrology of precision optical surfaces.

Optifab 2013, edited by Julie L. Bentley, Matthias Pfaff, Proc. of SPIE Vol. 8884,888413

(C) 2013 SPIE · CCC code: $0277-786 X / 13 / \$ 18 \cdot$ doi: $10.1117 / 12.2030508$ 
We introduce two improvements in the topographic optical profilometry by absorption in fluids (TOPAF) leading to a high reliability [16-18]. First, we use two narrow spectral bands for illuminating. In this way, we get an absorption signal and a reference signal without moving or changing anything in the setup. In addition, a constant ratio between the radiances of both spectral bands guarantees a non-distorted profile measurement. Secondly, we use extended light sources to reach high numerical apertures of illumination. This strategy allows measuring steep slopes $\left(<90^{\circ}\right)$ in a simple way.

In a previous work [18] we demonstrate the ability to measure steep slopes and we used laser sources to provide intrinsic linearity in the evaluation model. Now, we introduce as primary light source the output from a monochromator slit. This provides flexibility by choosing the strength of absorption without changing the fluid, and also it allows a way to minimize transverse chromatic aberration of the imaging system by bringing the lines closer. We present several analysis and results to show the metrological properties of TOPAF in terms of resolution, shape accuracy, its adaptability to any surface finish and independently of scale (aperture). We achieve a height resolution in the order of nanometers and similar shape accuracy. These features may be of interest for the measuring of precision polished or ground optical parts in a fabrication process.

\section{DESCRIPTION OF THE METHOD}

A reference surface is located close to the surface we want to profile (Fig. 1). An optical absorbing liquid or fluid fills the gap between both surfaces (sample and reference). Typically, it is a water soluble dye to simplify cleaning operations. This optical sandwich is situated between an extended light source and an imaging device that focus the surface to profile. The basic procedure consists on taking images at two narrow spectral bands centered at $\lambda_{A}$ and $\lambda_{R}$. They are selected so that the absorption of the dye is significantly greater at $\lambda_{A}$, and the band centered at $\lambda_{R}$ is used to provide a reference signal (Fig. 1b). The differential absorption between these bands provides direct information on the surface topography with respect to the reference.

b)
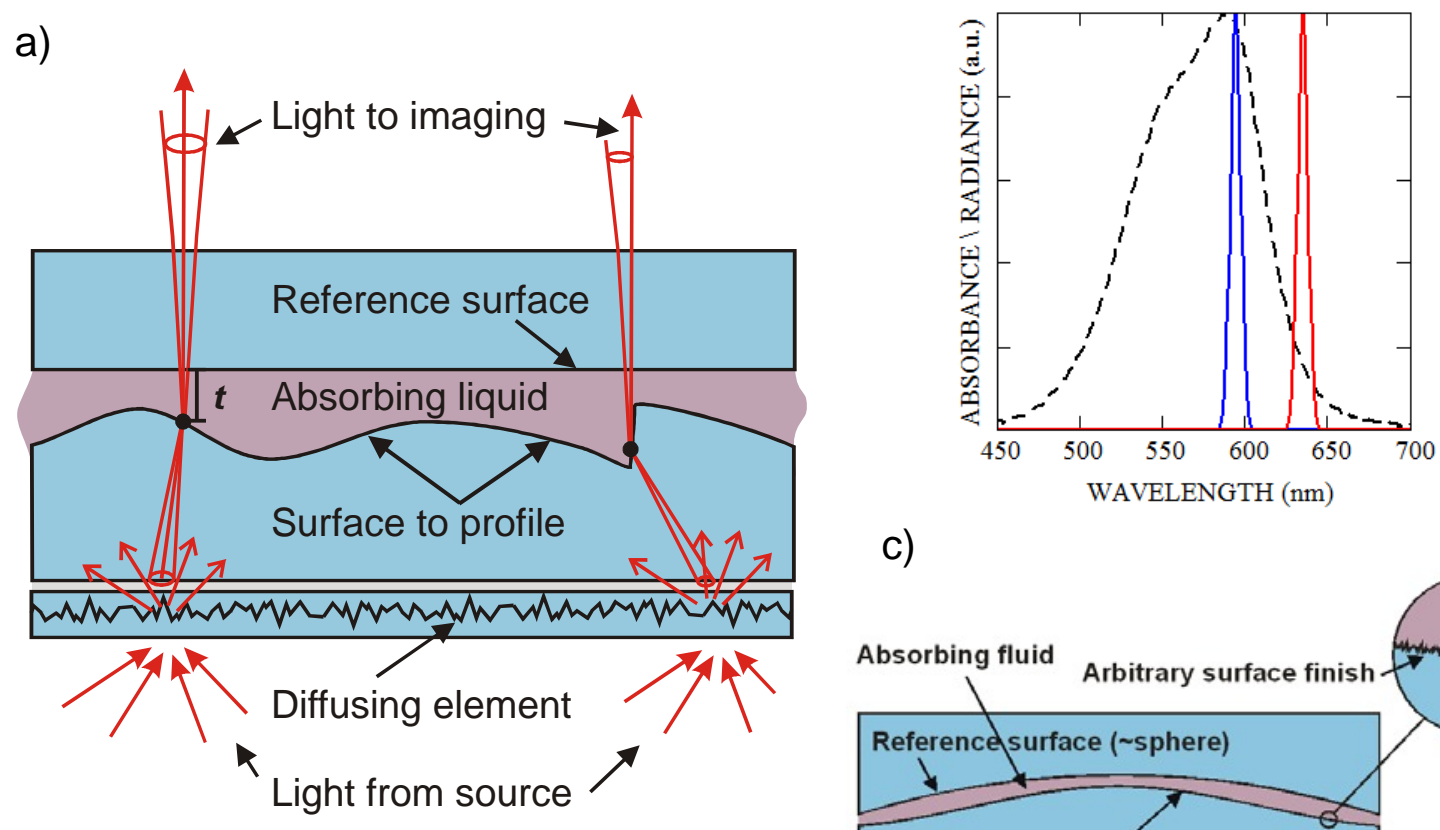

c)

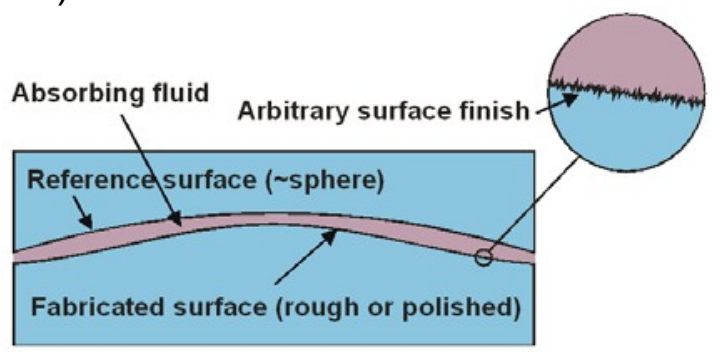

Fig. 1 a) TOPAF's basic setup. An absorbing water-soluble dye is sandwiched between two surfaces. A transmission image provides topographic data of the surface of interest by reference to the other surface. b) Absorption spectra of a water soluble dye (dash line) and the light source radiances used in measurements. c) Possible setup for aspheric or free-form metrology. Notice the measured surface can be either rough or polished. 
An extended light source provides wide spread angular illumination (for example an integrating sphere). A diffusing element optically coupled may also be used to increase the illuminating angular aperture (Fig. 1a). In this way, at every imaged spot of the surface to profile, we make sure that the light beam that comes from the substrate and propagates to imaging will reach and fill the entrance pupil of the imaging device, independently of ray refraction at slopes or the nature of the surface (rough or polished). For very steep slopes (near $90^{\circ}$ ) this is strictly true if the refractive index of the fluid is lower than the refractive index of the substrate of the surface to profile.

Consider the setup of Fig. 1a and assume near telecentric observation with small aperture (or big depth of focus) and that the fluid has a refractive index close to optically match the reference surface $(\Delta n<0.06)$. These conditions allow to: 1) simplify the tracing of principal rays and, 2) neglect multiple reflections within the fluid interfaces.

There are several ways to measure the transmittance within the liquid which is the basis for the profilometric probe. We describe next a preferred procedure based on the bi-chromatic illumination because of its simplicity and robustness. The imaging system provides an image proportional to the transmitted radiance $L$ of the sample and given as (LambertBeer law)

$$
L=L_{0} T \exp (-\alpha \cdot t),
$$

where $L_{0}$ is the radiance of the extended light source taken at the considered surface spot and line of sight (beam trajectory), $T$ is the total transmittance of the optical system except the fluid. The internal fluid transmittance is included in the exponential term, where $\alpha$ is the absorption coefficient of the fluid and $t$ is the distance from the reference surface to a sample surface spot as shown in Fig. 1a (consider it positive).

The extended light source below the substrate of Fig. 1a provides illumination at two different narrow spectral bands, centered at wavelengths $\lambda_{R}$ and $\lambda_{A}$. The absorption coefficients of the liquid for these wavelengths are $\alpha_{R}$ and $\alpha_{A}$ respectively (see Fig. 2). We define the measuring magnitude $M$ as the ratio of the "absorption" image taken at $\lambda_{A}$ to the "reference" image taken at $\lambda_{R}$, pixel by pixel, i.e. from (1):

$$
M=\frac{L_{0 A} T_{A} \exp \left(-\alpha_{A} \cdot t\right)}{L_{0 R} T_{R} \exp \left(-\alpha_{R} \cdot t\right)}=C \exp \left[-\left(\alpha_{A}-\alpha_{R}\right) \cdot t\right],
$$

where $C\left(C=L_{0 \mathrm{~A}} T_{\mathrm{A}} / L_{0 \mathrm{R}} T_{\mathrm{R}}\right)$ is a system factor that does not depend on the profile. This system parameter $C$ should be a spatial constant. For uniform transmittances at reference and sample substrates the ratio $T_{\mathrm{A}} / T_{\mathrm{R}}$ is a constant. The ratio $L_{0 \mathrm{~A}} / L_{0 \mathrm{R}}$ is also constant for Lambertian light sources, but not only. In fact, a non-uniform light source could be used if the ratio between radiances at the reference and absorbing spectral bands is constant. In other words, the radiance from the illuminating source has to be chromatically constant (same color) independently of surface point and angle of observation. In such a case, we may rewrite expression (2) as

$$
M=\exp \left[-\alpha_{\mathrm{S}}\left(t-t_{0}\right)\right]
$$

where $\alpha_{\mathrm{S}}=\alpha_{\mathrm{A}}-\alpha_{\mathrm{R}}$, and $t_{0}$ comes from rewriting $C$ as $C=\exp \left(\alpha_{\mathrm{S}} t_{0}\right)$. Finally, we can calculate the profile point by point from Eq. (3) by using the following form:

$$
t=t_{0}-\ln (M) \cdot t_{\mathrm{S}},
$$

where the characteristic distance $t_{\mathrm{S}}$ is related to the differential absorption $\alpha_{\mathrm{S}}$ as $t_{\mathrm{S}}=1 / \alpha_{\mathrm{S}}$. The parameter $t_{0}$ represents a baseline height or piston term that is constant under adopted assumptions.

The characteristic parameter of the fluid $t_{\mathrm{S}}$ defines the height range of the method. For the particular case of zero absorption at the reference wavelength $\left(\lambda_{R}\right)$, the parameter $t_{\mathrm{S}}$ represents the distance that the light at absorbing wavelength $\left(\lambda_{A}\right)$ propagates within the liquid until it attenuates to $\sim 37 \%(T=1 / \mathrm{e})$. As $t_{\mathrm{S}}$ depends on absorption strength, it can be set to different values by varying the concentration of the dye or the couple of wavelengths $\lambda_{R}, \lambda_{A}$. The achievable height resolution and the height range are linked to $t_{\mathrm{S}}$ in a fundamental way as we will see next. 


\section{EXPECTED PERFORMANCE}

The expected height sensitivity ( $\Delta t)$ can be estimated from Eq. (4) as

$$
\Delta t=-(\Delta M / M) \cdot t_{\mathrm{S}},
$$

which conveniently express a small height variation $\Delta t$ in terms of a small relative variation of the measured ratio $(\Delta M / M)$ and the characteristic distance of the absorbing fluid $t_{\mathrm{S}}$. The ultimate resolution of a measurement depends basically on the signal to noise ratio (SNR) of the imaging device. It can be defined as SNR $=N / \Delta N$ where $N$ is the signal reading at CCD (expressed in gray levels or counts $N$ and proportional to the radiance $L$ ) and $\Delta N$ is the final random noise. For a scientific CCD camera, SNR can be typically estimated as the square root of the electron well depth at best. Typical values for the SNR of a single pixel signal are between 40:1 and 120:1. Usually we take a maximum of 4 different images (with the background subtracted): a minimum of 2 of them for the estimation of the absorption and reference and the other 2 for calibration of the photo-response non-uniformity (PRNU) of the CCD sensor. This calibration is necessary to make the piston term $t_{0}$ really a constant and not dependent on a non-uniform response of the CCD. This four image processing leads to a final uncertainty for $M$ of $\Delta M / M=2 / S N R$, i.e. the best height resolution $\Delta t$ is about a $\sim 2 \%$ of $t_{\mathrm{S}}$ in a single shot acquisition and per pixel. Multiple frame integration and/or spatial pixel averaging can increase SNR and therefore height resolution in one or two orders of magnitude as we will see in the experimental results. In general terms, a final height resolution better than $10^{-3} t_{\mathrm{s}}$ can be reasonably expected. We may find highly absorbing substances with $t_{\mathrm{S}}$ values even below 1 micron for concentrated solutions. According to the previous analysis this means that nanometer-level height resolution is achievable.

The photometric dynamic range of the imaging sensor determines the height profile range of the method. In practice, the height range is not much bigger than $t_{\mathrm{S}}$ because of the exponential decay of transmission. For example, at a depth of $4 t_{\mathrm{s}}$ the transmittance is reduced to a mere $\sim 2 \%$. Notice that both height resolution and height dynamic range are linked to the fluid parameter $t_{\mathrm{S}}$ and this parameter can be tailored by means of the dye concentration or the couple of wavelengths chosen: $\lambda_{R}, \lambda_{A}$.

From a metrological point of view, we have considered other uncertainty and error sources to take into account in the experimental results and that may affect shape accuracy specially. For shake of brevity, we leave for another publication the specific consideration of these corrections. All of the following contributions have been evaluated, controlled or minimized by calibration:

- Spatial coordinates calibration: imaging magnification, distortion and chromatic aberration included.

- Requirement of constant chromatic radiance ratio for the light source.

- Non-linearity due to CCD response and to the effect of finite spectral bandwidth of illumination.

- Environment: relative humidity and temperature changes lead to $t_{\mathrm{S}}$ variations within the fluid.

- Background signal including CCD dark signal and scattering within optics.

- Internal and external multiple reflections.

- Lack of telecentrism or ray-trace errors.

- Cleaning of optics (invisible residues lead to profile errors typically in the range of nanometers).

For the experimental setup we used, the combined contribution of all these uncertainty sources (after correction) to the final uncertainty of estimated height or sag is in the range of $\sim \pm(0.1 \%-0.4 \%)$ of $t_{\mathrm{S}}$ for the considered cases.

\section{RESULTS}

We implement the setup as shown in Fig. 2. An integrating sphere acts as extended uniform light source (Fig. 2a). It was internally illuminated from the output slit of a single monochromator, providing two alternate beams, one at $\lambda_{A}=594 \mathrm{~nm}$ and the other at $\lambda_{R}=635 \mathrm{~nm}$ (bandwidth of $9 \mathrm{~nm}$ FWHM). These beams impinge on the wall of the sphere opposite to the input port of the integrating sphere. The equal geometrical propagation for both beams (reference and absorption) is fundamental to achieve a constant chromatic radiance ratio. Finally, an opal diffusing glass is situated at the output port to further homogenize the light source before the sample. In experiments we arrange the reference first and then the surface to measure. For the considered cases this arrangement does not affect the previous analysis (Fig. 2a). The spectral bands have been chosen to provide minimum chromatic aberration at the images, maximum thickness sensitivity 
and, at the same time, good linearity performance. Finite bandwidths and sloping absorbance curves lead to non-linearity in the thickness estimation, i.e., Eq. (4) is strictly valid for monochromatic light sources or for flat absorbance curves within bandwidth. However, a careful choice of band positions and bandwidths can auto-compensate this non-linearity effect. We check it by simulations and in practice by measuring a wedge of flats (we will see it later).

As absorbing dye we used methyl violet at different concentrations in a mixture of water and glycerol and filtered to $0.8 \mu \mathrm{m}$ particles. The glycerol-water mixture has proven adequate to avoid evaporation/condensation of water that would change absorption properties. The ambient at the lab were typically at $\mathrm{T}=30 \pm 2^{\circ} \mathrm{C}$ and at a relative humidity of $45 \pm 3 \%$. The absorption spectrum of the fluid and the illuminating bands are shown in Fig. 1b. The fluid mixtures typically we used in experiments had $t_{\mathrm{S}}$ from $\sim 5 \mu \mathrm{m}$ to $\sim 80 \mu \mathrm{m}$ and a refractive index near 1.46 for $\lambda=630 \mathrm{~nm}$. Most of the processed images integrate 128 frames taken within less than a half a minute and with a spatial averaging of 3 pixels to improve height resolution. Other setup characteristics and experimental details can be found in [16].

a)

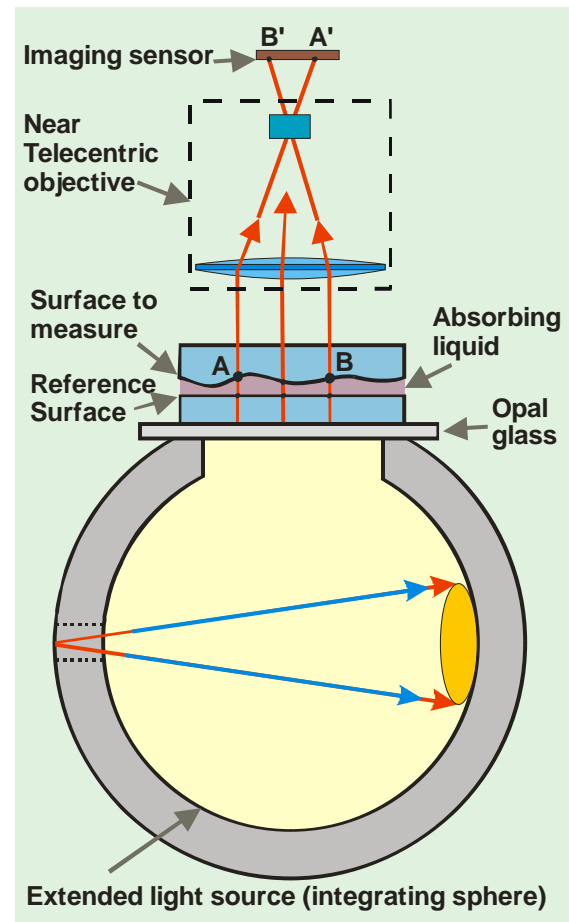

b)

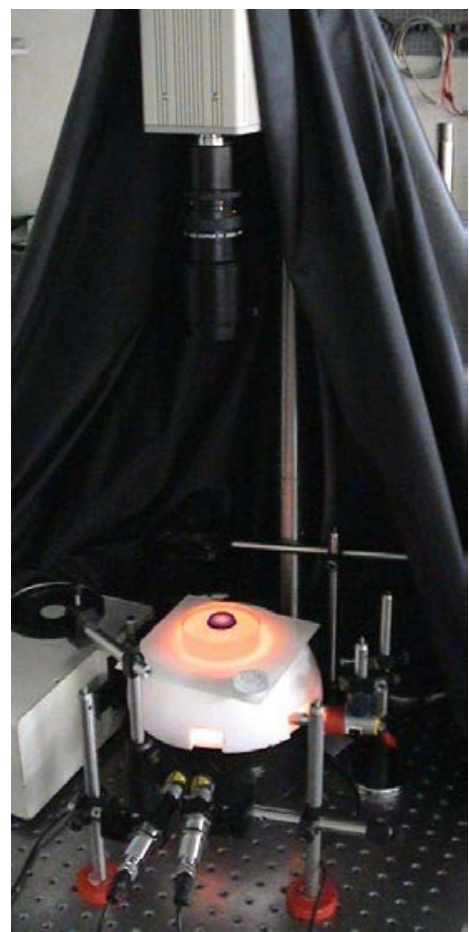

Fig. 2 (Color online) Complete setup considering the imaging system and the light source. a) Diagram of principal parts. b) Picture of the setup at the lab. An obscuration curtain encloses the setup when measuring to avoid background light.

In Fig. 3 we show a check for the necessary condition of a constant chromatic ratio of radiances at the light source. We got images of the light port at both spectral bands $\left(\lambda_{A}, \lambda_{R}\right)$ and corrected of CCD PRNU, therefore proportional to the radiance at these bands. We show them and its ratio in Fig. 3a. The considered light port is an optical flat matched underneath to an opal glass lying onto the 4" aperture of the integrating sphere. While the observed monochromatic radiances are spatially non-uniform, around a $\pm 10-20 \%$ (color thin lines in the linear plot of Fig. 3b), the ratio between these radiances is basically constant. This ratio deviates less than a $\pm 0.1 \%$ within the region of interest (black bold line in the linear plot of Fig. 3b). This improvement of two orders of magnitude in the uniformity demonstrates the usefulness of using the chromatic ratio instead of the direct radiances and guarantees that the piston term $t_{0}$ in Eq. (4) is really a constant.

A first test was performed to validate Eq. (4) under the described illuminating setup. Two optical flats were arranged to make a thin wedge prism filled with a fluid absorber of $t_{\mathrm{S}}=29.4 \mu \mathrm{m}$ (Fig. 4a) and a maximum height of $\sim 70 \mu \mathrm{m}$. The surface accuracy of one flat is $\lambda / 20$ and the other is $\lambda / 10$. We obtained the processed 3D-profile image of Fig. 4b. A linear profile of this image is extracted in Fig. 4c that qualitatively illustrates good performance. A quantitative regression fit to a plane was performed for the topographic map of Fig. 4b. The residuals of the fit are shown in Fig. 4d. We obtained a RMS of $\pm 59 \mathrm{~nm}$ over a $90 \%$ of the aperture. This result is coherent with the nominal surface accuracy of the wedge. 
a)

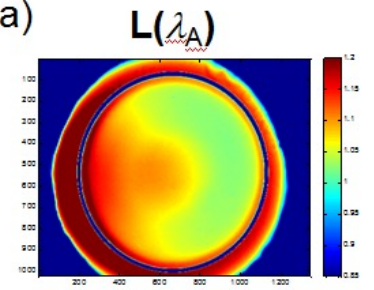

b)

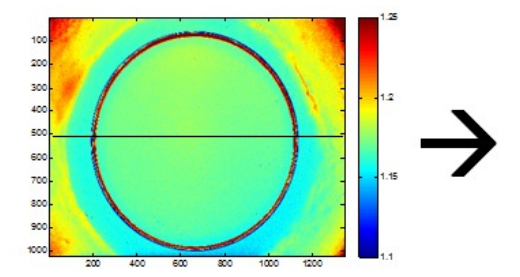

$L\left(\lambda_{R}\right)$
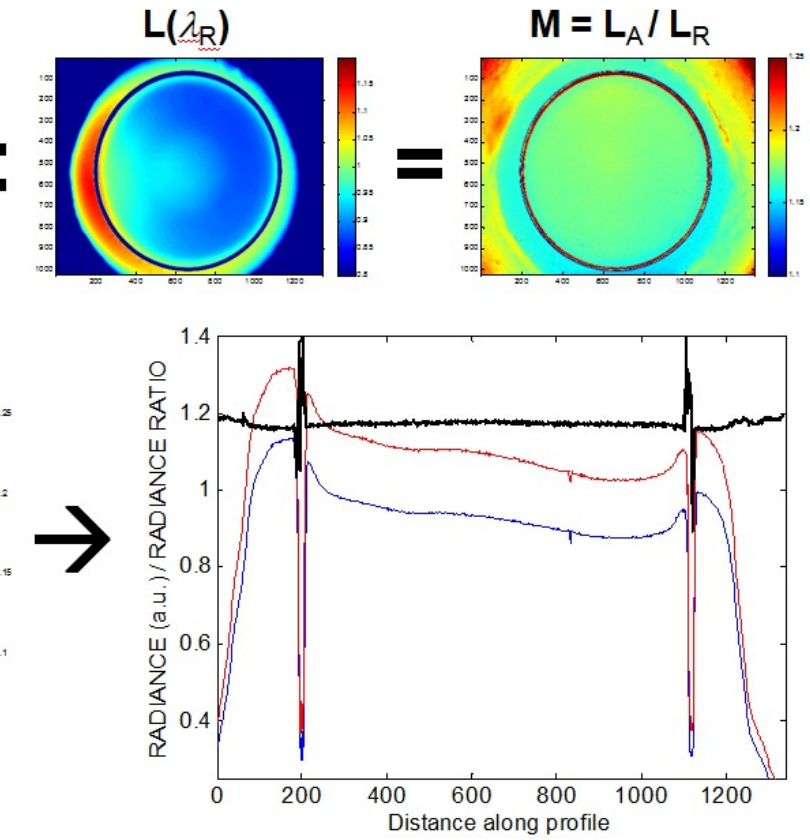

Fig. 3 (Color online) a) Images of the light source port representing the monochromatic radiances at $\lambda_{A}$ and $\lambda_{R}\left(L_{A}, L_{R}\right)$ and the chromatic radiance ratio. b) Linear profile of the radiances and its ratio. The color lines represent the radiance profile at every spectral band and the black bold line represents the profile along its ratio.

a)

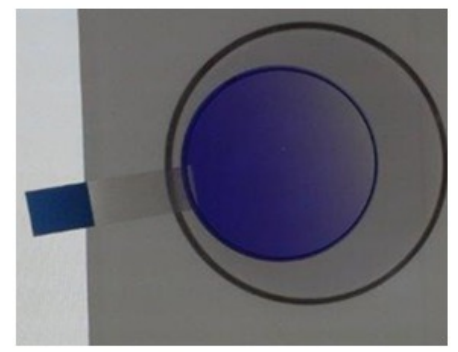

c)

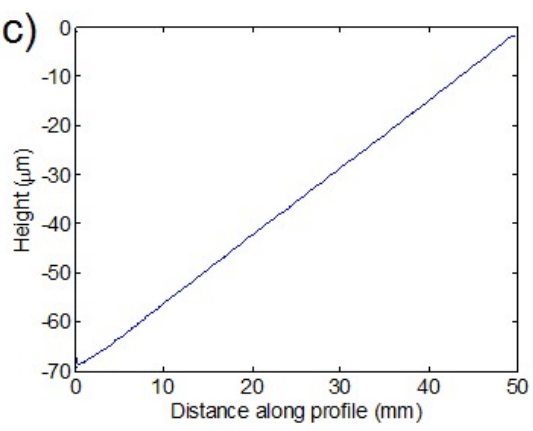

b)

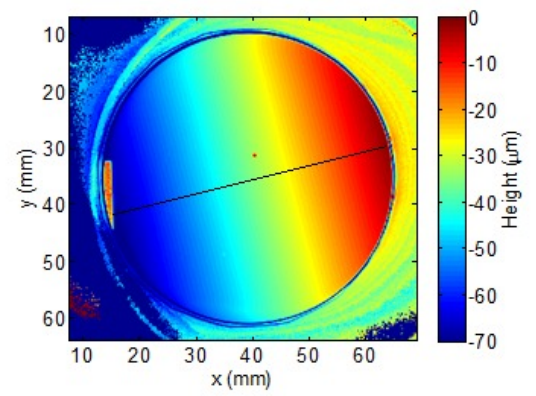

d)

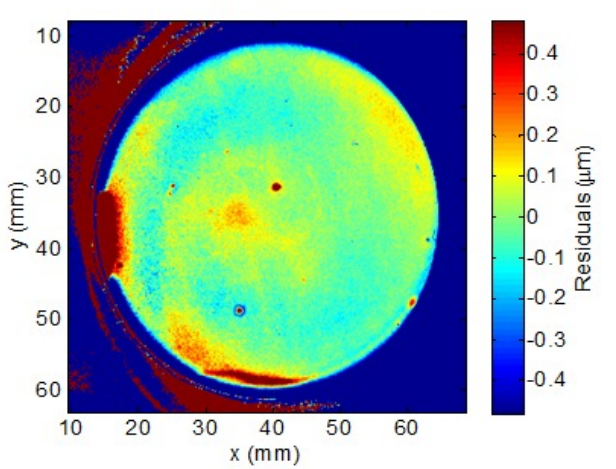

Fig. 4 (Color online) a) Sample preparation for a wedge of flats. b) Thickness evaluation image after Eq. (4). c) linear profile extracted from b). d) Regression fit to a plane for the topographic image of b). A fit RMS of $\pm 59 \mathrm{~nm}$ is obtained over a $90 \%$ of aperture.

We also tested the capability of the method to measure polished or rough surfaces indistinctly. To get a rough finish we apply a chemical paste used in graphical arts to give a matte finish at glass surfaces. We etched one side of a 1" window of B270 glass over a crossing strip. The visual appearance of the result is shown in the Fig. 5a. The treated side 
was placed against a $\lambda / 10$ flat surface as reference. The absorbing fluid filling the gap had a $t_{\mathrm{S}}=4.65 \mu \mathrm{m}$ (see Fig. $5 \mathrm{~b}$ ). We performed a topographic measurement on the whole aperture showing the final result in Fig. $5 \mathrm{c}$ with tilt removal. Lateral scale resolution of the image represents around $70 \mu \mathrm{m}$ per pixel. A linear profile is extracted from it and shown in the Fig. 5d. Finally, a 3D plot (partial view) may be seen in the Fig. 5e. A regression fit to a plane shows for the polished part a local RMS of $\pm 7 \mathrm{~nm}$. At the rough strip the RMS is of $\pm 178 \mathrm{~nm}$ (whole strip).

a)

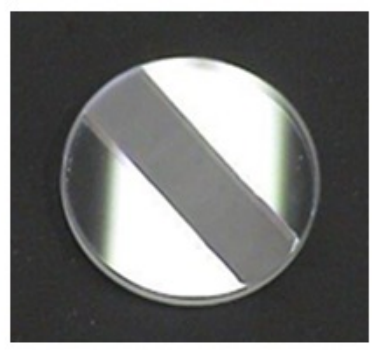

b)

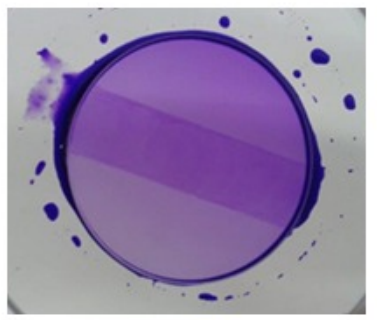

c)

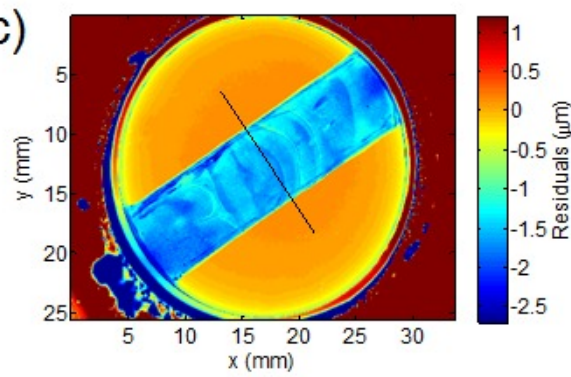

d)
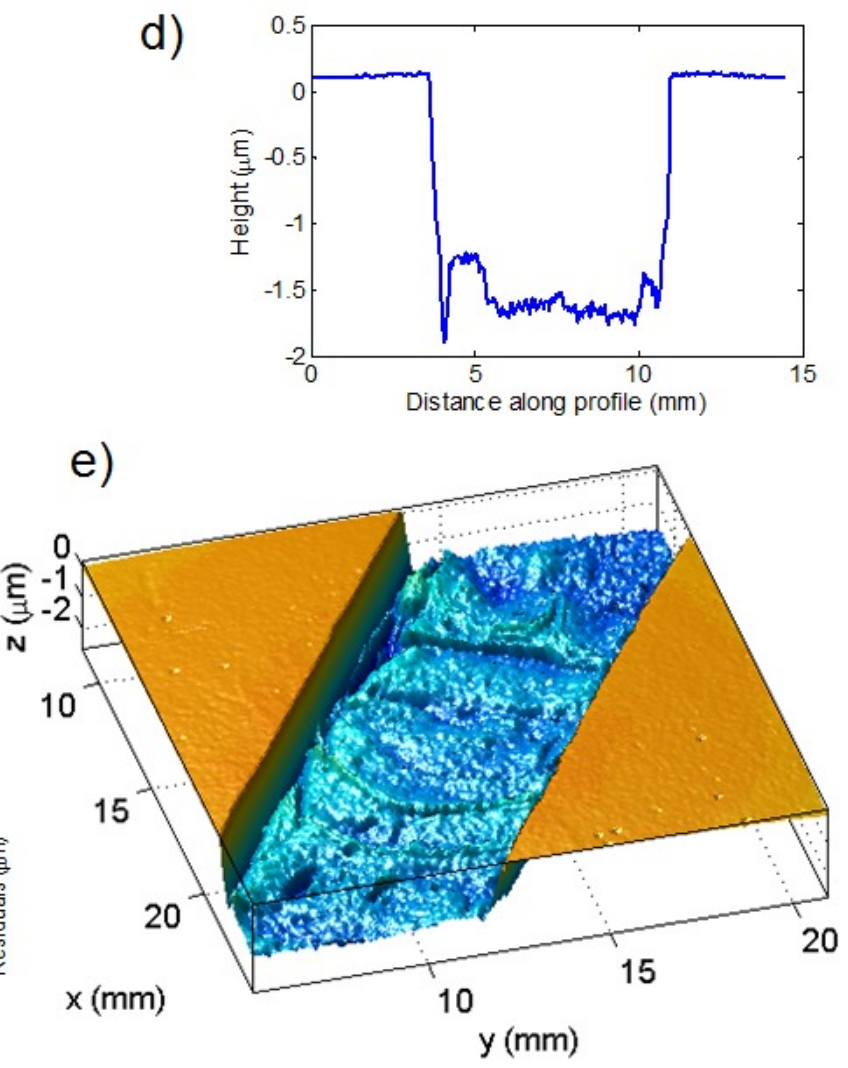

Fig. 5 (Color online) Topographic measurements on a flat surface with polished and rough areas. a) Natural appearance of a window (B270 glass) with a rough strip in one side made by chemical etching. b) Sample preparation with absorbing fluid. c) Processed topographic image with tilt removal. d) Linear profile extracted from c). e) 3D plot of a partial view of c).

Next we show some experimental results concerning the shape accuracy of the method. We used sphere surfaces with a surface accuracy of $\lambda / 10$ (Fig. 6 and Fig. 7) that belong to lens substrates of NBK7 glass. In the first case we used a plano-convex lens of $25.4 \mathrm{~mm}$ of aperture with a sphere surface of nominal radius of curvature of $5151 \mathrm{~mm}$. In the experimental setup this surface was referenced to a $\lambda / 20$ flat surface of silica substrate (as illustrated in Fig. $6 \mathrm{~b}$ ). The absorbing fluid had a $t_{\mathrm{S}}=4.73 \mu \mathrm{m}$. Expected maximum sag deviation for this setup is around $15.5 \mu \mathrm{m}$. In Fig. 6a we show the original chromatic images and its ratio. After processing with Eq. (4) a regression fit to a best sphere over the $80 \%$ of the aperture gives the residuals image of Fig. 6c. The RMS deviation is of $\pm 35 \mathrm{~nm}$ (5 pixel spatial averaging). When the regression fit is restricted to a central $30 \%$ of the aperture it gives a RMS of $\pm 7 \mathrm{~nm}$. These results are in agreement with the nominal surface accuracy. A linear profile is extracted from the residuals map and shown in Fig. 6d.

In the second case we used a plano-convex lens of $38.1 \mathrm{~mm}$ of aperture with a sphere surface of nominal radius of curvature of $257.5 \mathrm{~mm}$. In the setup it was referenced to a concave sphere of nominal radius of $309.1 \mathrm{~mm}$ from a planoconcave lens of $50.8 \mathrm{~mm}$ of aperture, as shown in Fig. 7a. The absorbing fluid filling the gap has now a $t_{\mathrm{S}}=43.8 \mu \mathrm{m}$. Maximum sag deviation should be around $118 \mu \mathrm{m}$ in this case. In Fig. 7b we show a topographic map after processing the measured images according to Eq. (4). We show in Fig. 7c a linear profile extracted from this map. A regression fit to a best sphere shape over the $80 \%$ of the aperture gives the residuals image of Fig. $7 d$ (RMS deviation of $\pm 0.127 \mu \mathrm{m}$ ). 
a)
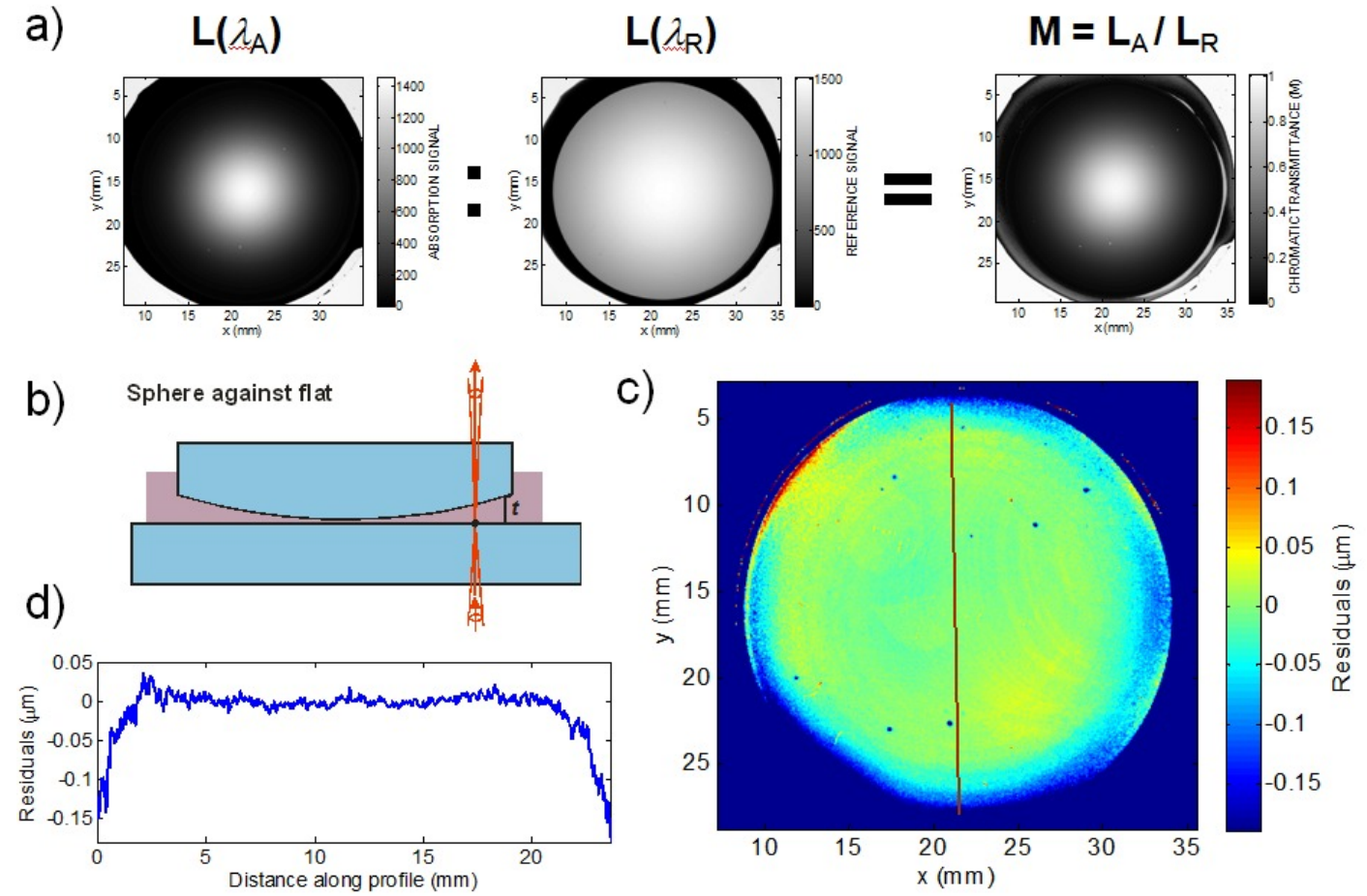

Fig. 6 (Color online) Measurement and analysis of a convex sphere surface (R=5151 mm, D=25.4 mm) referenced to a flat surface. a) Images taken at every spectral band $\left(\lambda_{A}\right.$ and $\left.\lambda_{R}\right)$ and its ratio $(M)$ which is introduced in Eq. (4) to estimate sag $t$. b) scheme of the sample setup. c) Residuals of the regression fit to a best sphere (in microns). d) Linear profile extracted from the residuals of c) (vertical line crossing the aperture).

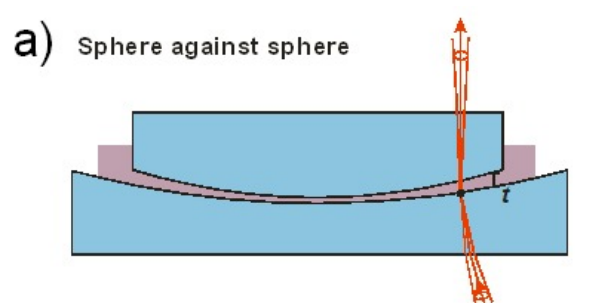

b)

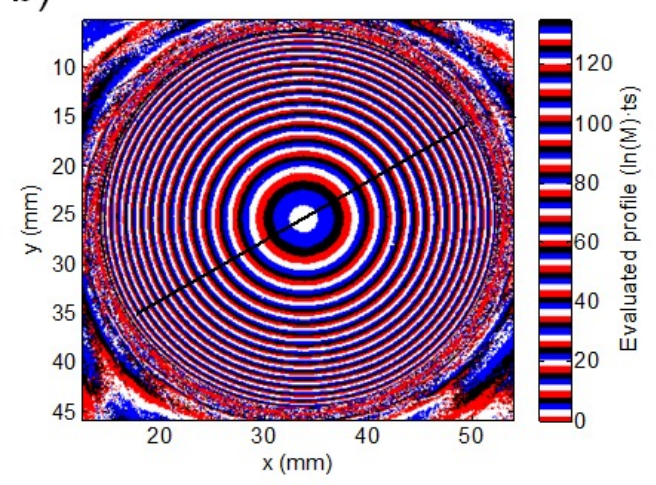

c)

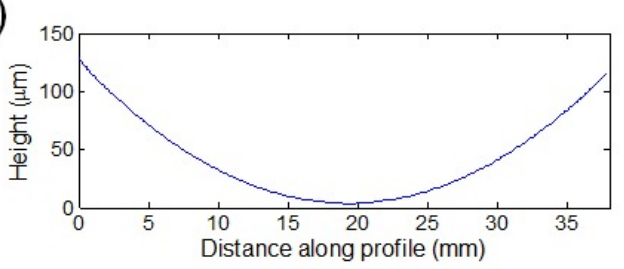

d)

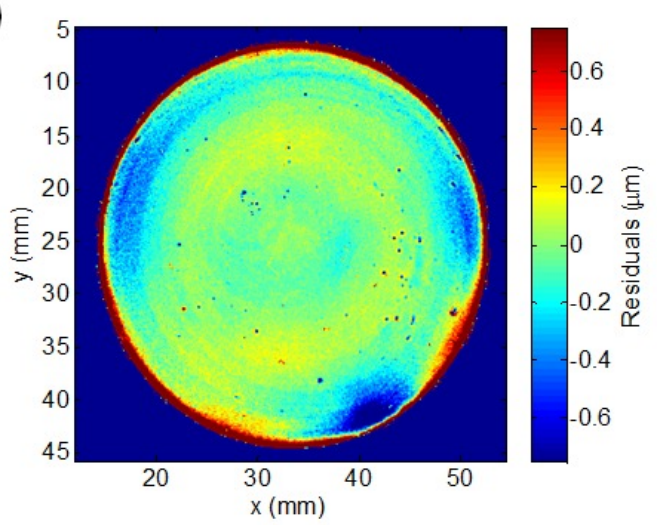

Fig. 7 (Color online) Measurement and analysis of a convex sphere surface ( $\mathrm{R}=257.5 \mathrm{~mm}, \mathrm{D}=38.1 \mathrm{~mm}$ ) referenced to a concave sphere $(R=309.1 \mathrm{~mm}, \mathrm{D}=50.8 \mathrm{~mm})$. a) Scheme of the sample-reference optical setup. b) Topographic map after processing the chromatic image ratio $M$ after Eq. (4). c) Linear profile extracted from b) representing sag $t$. d) Residuals of the regression fit to a best sphere (in microns). 
a)

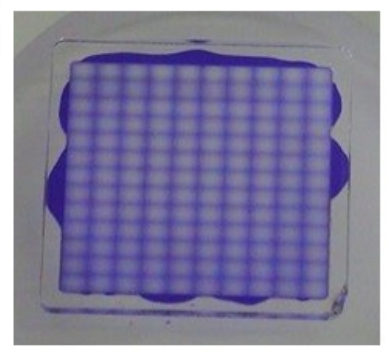

b)

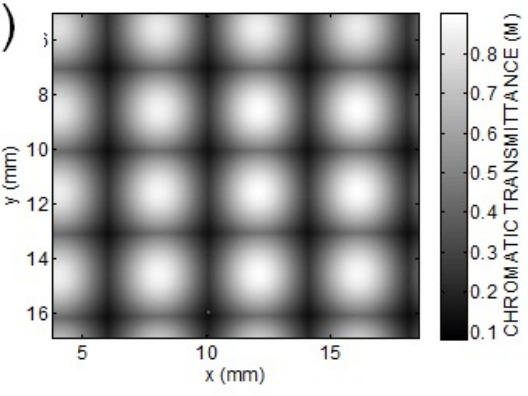

C)

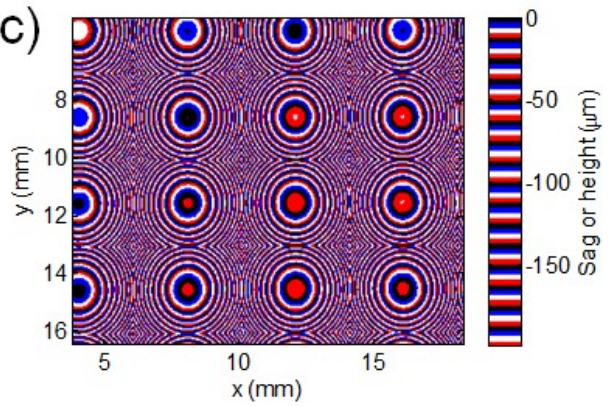

d)

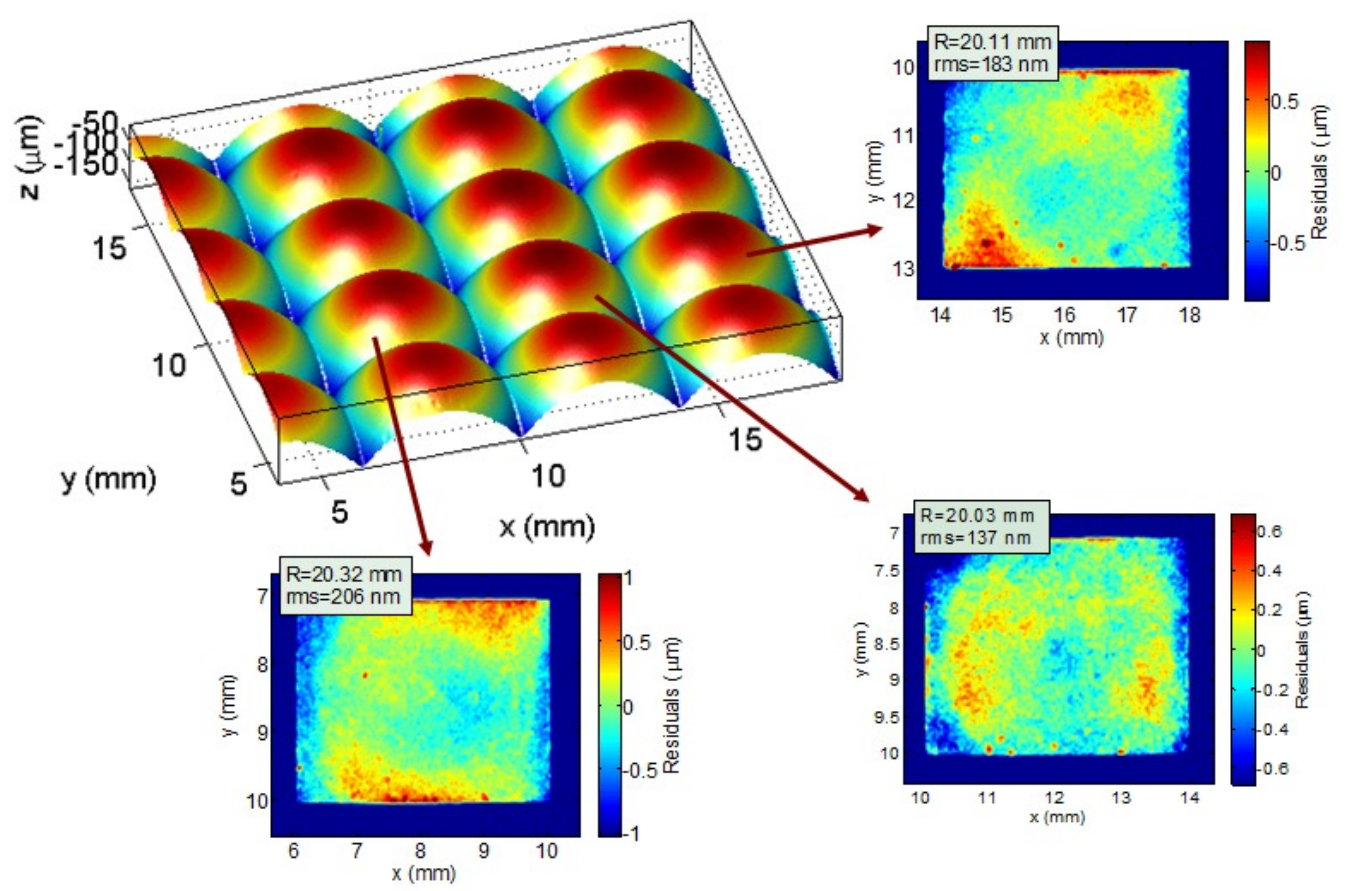

Fig. 8 (Color online) Topographic characterization of a spherical lens array. a) Appearance of the prepared sample. b) Image of the chromatic transmission ratio $\mathrm{M}$ (partial view). c) Processed topographic image with a fringe-like representation. d) $3 \mathrm{D}$ plot of c). To the right-below some inserts show the residuals and the calculated radius of the regression fit to a best sphere for some lens elements.

Finally, as a representative of optical surfaces with complex shapes, we show the results obtained for a multi-lens array in Fig. 8. The individual spherical lenses have nominal radius of $20 \mathrm{~mm}$, an aperture of $4 \times 3 \mathrm{~mm}$ and they are AR coated on both sides. Maximum sag deviation should be around $156 \mu \mathrm{m}$ in this case by reference to a flat. In Fig. 8a we show the sample prepared with a fluid dye of $t_{\mathrm{S}}=82.54 \mu \mathrm{m}$ and referenced to a flat surface $(\lambda / 10)$ on a silica substrate. In Fig. 8b we show a partial view of the measuring magnitude $M$ from which we get the topographic map according to Eq. (4) and shown in Fig. 8c. Finally, we show in Fig. 8d a 3D-plot and include the residuals of the regression fits to a best sphere for some lenses within the array. Typical RMS is around $\pm 170 \mathrm{~nm}$ and the estimated radius of curvature and its variability is in agreement with the nominal data $(\mathrm{R}=20 \mathrm{~mm} \pm 2 \%)$.

This last case may be used to illustrate some differences with an interference method. If a conventional interferometric measurement proceeds with a plane wavefront as a reference, then the fringe density will be too high within the field of view. This fact may be appreciated in Fig. 8c where a fringe-like representation of the topography has been chosen for this purpose. If we choose a spherical wavefront as a reference we only can measure a single lens at a time instead of the full field of view possible with TOPAF. Finally, we also applied the presented method to characterize some aspheric lenses and free-form ophthalmic lenses. However, the lack of appropriate reference data on these surfaces to compare with has prevented us to publish the results. In this sense we encourage the scientific and industrial community to establish a collaboration environment where these and other metrological possibilities may develop further. 
In this contribution we have shown some of the possibilities of TOPAF's imaging method to measure the shape of optical surfaces at the level of precision optics. We have also shown its capability to handle both polished and rough surface finish with the same setup. It is intrinsically not limited by the form, slope and the optical aperture or lateral scale. The sag or height resolution is decoupled from lateral spatial resolution. Therefore, this method seems especially appropriate for the metrology of all type complex surface shapes (on transparent substrates), including polished and rough finished parts.

\section{REFERENCES}

[1] Thompson, K.P., and Rolland, J.P., “A revolution in imaging optical design,” Opt. Phot. News 23, 31-35 (2012)

[2] Cheng-Chung Lai and I-Jen Hsu, "Surface profilometry with composite interferometer", Opt. Express 21, 13949-13955 (2007)

[3] Deck, L., de Groot, P., "High-speed noncontact profiler based on scanning white-light interferometry", Appl. Opt. 31, 7334-7338 (1994)

[4] Wyant, J.C. “Advances in interferometric surface measurement”, Proc. SPIE 6024, 602401-11, (2005)

[5] Chenguang Zhao, Jiubin Tan, Jianbo Tang, Tao Liu, Jian Liu, "Confocal simultaneous phase-shifting interferometry”, Appl. Opt. 50, 655-661 (2011)

[6] Chau-Hwang Lee, Hong-Yao Mong, and Wan-Chen Lin, "Noninterferometric wide-field optical profilometry with nanometer depth resolution," Opt. Lett. 27, 1773-1775 (2002)

[7] Blais, F., "Review of 20 years of range sensor development”, J. Elect. Imag. 13, 231-243 (2004)

[8] Chen, F., Brown, G.M., Song, M., “Overview of three-dimensional shape measurement using optical methods”, Opt. Eng. 39, 10-22 (2000)

[9] Purcell, P., Suratkar, A., Davies, A., Farahi, F., Ottevaere, H., Thienpont, H., "Interferometric technique for faceted microstructure metrology using an index matching liquid”, Appl. Opt. 49, 732-738 (2010)

[10] Svensson, G., "A method for measurement of the absorption in extremely high-absorbing solutions", Experimental Cell Research 9, 428-133 (1955)

[11] Csete, M., Bor, Zs., "Plano-concave microcuvette for measuring the absorption coefficient of highly absorbing liquids”, Appl. Opt. 36, 2133-2138 (1997)

[12] Johnson, J., Harris, T. "Full-field optical thickness profilometry of semitransparent thin films with transmission densitometry”, Appl. Opt. 49, 2920-2928 (2010).

[13] Ogilvie, S., Isakov, E., Taylor, C., Glover, P., "A new high resolution optical method for obtaining the topography of fracture surfaces in rocks”, Image Anal. Stereol. 21, 61-66 (2002).

[14] Isakov, P.E., Ogilvie, S.R., Taylor, W.C., Glover, P.W.J., "Fluid flow through rough fractures in rocks I: high resolution aperture determinations", Earth and Planetary Science Letters 191, 267-282 (2001).

[15] Model, M.A., Khitrin, A. K., Blank, J.L., "Measurement of the absorption of concentrated dyes and their use for quantitative imaging of surface topography”, J. Microscopy. 231, 156-167 (2008)

[16] Martinez Antón, J. C., "Three-dimensional profilometer based on optical absorption in fluids", patent application WO2013011172 (2011).

[17] Martinez Antón, J. C., Alonso Fernández, J., Gómez Pedrero., J. A., and Quiroga, J. A., “Optical method for the surface topographic characterization of Fresnel lenses,” Proc. of SPIE 8169, 816910-1 (2011).

[18] Martinez Antón, J. C., Alonso, J., Gómez Pedrero., J. A., and Quiroga, J. A., “Topographic optical profilometry by absorption in liquids”, Opt. Exp. 20(27), 28631-28640 (2012). 